\title{
Manejo ambulatorio de la neumonía comunitaria del adulto en las unidades de emergencia. Servicio de Salud Viña del Mar-Q uillota de la V Región
}

\author{
Juana Pavié $\mathrm{G}^{1}$, Julio Manuel de la Prida $\mathrm{C}^{2}$, Alejandro \\ Díaz $\mathrm{F}^{3}$, Fernando Saldías $\mathrm{P}^{3}$. \\ Assessment of the management of \\ community-acquired pneumonia in \\ adults outpatients
}

Background: There is limited information about the effectiveness of the treatment of community-acquired pneumonia (CAP) in Chilean emergency rooms. Aim: To assess the treatment of CAP in emergency rooms at the Viña del Mar Health Service in Chile. Material and methods: Prospective study of immunocompetent adult patients consulting for a CAP in emergency rooms. Those that required hospital admission were considered ineligible. The initial clinical and laboratory assessment, antimicrobial treatment and their condition after 30 days of follow up, were recorded. Results: Three hundred eleven adult patients aged $57 \pm 22$ years ( 152 males), were evaluated. Patients with class I CAP ( $40 \%$ of cases) were treated with Clarithromycin (71.8\%) or Amoxicillin (26.6\%) for 10 days. Patients with class II CAP (60\%) were treated with Amoxicillin-clavulanate (80.7\%) or Levofloxacin (18.2\%) for 10 days. Three hundred eight patients (99\%) were cured without need of hospital admission; three patients (1\%) were subsequently hospitalized because of clinical failure of ambulatory treatment. Overall, three patients (1\%) died; all deaths occurred during or immediately after hospitalization and were related to the severity of lung infection but not to the choice of antibiotic treatment. Conclusions: The outpatient management of CAP by general practitioners working at emergency rooms was clinically effective with low rates of hospital admission and mortality (Rev Méd Chile 2005; 133: 1322-30).

(Key Words: Anti-bacterial agents; Frail elderly; Pneumonia)

Recibido el 8 de marzo, 2005. Aceptado el 16 de junio, 2005.

${ }^{1}$ Servicio de Medicina del Hospital de Quillota. ${ }^{2}$ Programa de Enfermedades Respiratorias del Adulto del Servicio de Salud Viña del Mar-Quillota. ${ }^{3}$ Departamento de Enfermedades Respiratorias de la Pontificia Universidad Católica de Chile. Santiago de Chile.

Correspondencia a: Dr. Fernando Saldías P. Departamento de Enfermedades Respiratorias, Pontificia Universidad Católica de Chile. Teléfonos: 6331541-3543242. Fax: 6335255. Marcoleta 345 - 4o Piso. Santiago, Chile. Email: fsaldias@med.puc.cl 
L a neumonía adquirida en la comunidad (NAC) constituye una causa frecuente de consulta ambulatoria y prescripción de antimicrobianos en la población adulta de los países desarrollados y en vías de desarrollo1,2. A partir de la década 1980-89, la letalidad de la neumonía comunitaria del adulto ha aumentado progresivamente, a pesar de la mayor disponibilidad de antibióticos de amplio espectro, nuevas técnicas de diagnóstico microbiológico y avances en el cuidado de los enfermos ${ }^{3,4}$. En Chile, la neumonía en sus diferentes categorías diagnósticas constituye la cuarta causa específica de muerte, en ambos sexos, con una tasa de mortalidad de 21,2 por 100.000 habitantes en el año $2002^{5}$. Una vez establecido el diagnóstico de neumonía, la evaluación de la gravedad del caso individual permite decidir el lugar de manejo (ambulatorio o en el hospital) y la elección del tratamiento antimicrobiano empírico, ya que las limitaciones de las técnicas de diagnóstico microbiológico impiden conocer el agente causal en la mayoría de los episodios. La neumonía comunitaria representa un importante problema de salud pública, considerando su elevada prevalencia, demanda de recursos de salud y letalidad.

Con el propósito de racionalizar la distribución de los recursos sanitarios siempre insuficientes para satisfacer la demanda de atención y sistematizar el manejo de la neumonía comunitaria, la Sociedad Americana de Tórax (ATS), la Sociedad Británica de Tórax (BTS) y la Sociedad Chilena de Enfermedades Respiratorias (SER) han publicado recomendaciones clínicas para la evaluación, diagnóstico y tratamiento antibiótico de la neumonía adquirida en la comunidad en el adulto inmunocompetente ${ }^{6-}$ ${ }^{8}$. Sin embargo, las recomendaciones de los expertos suelen estar basadas, principalmente, en estudios clínicos realizados en pacientes con factores de niesgo y gravedad hospitalizados por neumonía comunitaria, siendo escasa la información disponible sobre el manejo ambulatorio de los enfermos.

En términos prácticos, los enfermos pueden ser estratificados en dos categorías de riesgo principales: a) pacientes sin criterios de gravedad que pueden ser manejados en el medio ambulatorio con antibióticos orales y tienen bajo riesgo de complicaciones y muerte (letalidad inferior a 1$2 \%)$; y b) pacientes con factores de riesgo de complicaciones y muerte (letalidad: 5-20\%) que deben ser admitidos al hospital con el propósito de proporcionarles todos los recursos disponibles para que logren superar la infección aguda ${ }^{9,10}$. Se ha estimado que entre $60 \%$ y $80 \%$ de los episodios de NAC son manejados en el medio ambulatorio, existiendo gran variabilidad en la tasa de hospitalizaciones en distintas áreas geográficas ${ }^{11-13}$. No disponemos de información actualizada sobre el manejo ambulatorio de la neumonía comunitaria del adulto inmunocompetente en nuestro medio. El propósito de este estudio es describir las características clínico-epidemiológicas y el manejo ambulatorio de la neumonía comunitaria del adulto inmunocompetente sin criterios de gravedad en las Unidades de Emergencia del sistema público de la V Región.

\section{PACIENTES Y MÉTODO}

Se evaluaron en forma prospectiva 311 adultos inmunocompetentes, atendidos en las Unidades de Emergencia de ocho centros asistenciales del Servicio de Salud Viña del Mar-Quillota por un episodio de neumonía comunitaria, entre el $1^{\circ}$ de enero y 31 de diciembre de 2003. Se incluyeron en el estudio los pacientes inmunocompetentes mayores de 15 años que cumplieron los criterios diagnósticos de neumonía comunitaria de Fang y cols $^{14}$ y los criterios de manejo ambulatorio sugeridos por la Sociedad Chilena de Enfermedades Respiratorias (SER) ${ }^{8}$. La neumonía se definió como un cuadro de evolución aguda de síntomas respiratorios (tos, expectoración mucopurulenta, disnea, dolor torácico), fiebre y la presencia de un infiltrado nuevo en la radiografía de tórax, de acuerdo a la evaluación del médico de urgencia. Se excluyeron del estudio los pacientes portadores del virus de inmunodeficiencia humana (HIV), tumores sólidos o hematológicos en quimioterapia, neutropenia (leucocitos $<1.000 / \mathrm{mm}^{3}$ ), tratamiento quimioterápico o inmunosupresor en los seis meses previos a la consulta.

El objetivo primario del estudio fue describir el manejo ambulatorio de los pacientes con NAC atendidos en la Unidad de Emergencia; así los pacientes admitidos al hospital por presentar criterios de gravedad ${ }^{6-8}$ fueron excluidos del protocolo, lo mismo ocurrió con aquellos que no tenían radiografía de tórax o ésta fue interpretada como normal. 
En los pacientes ingresados al protocolo, se registraron los siguientes antecedentes clínico-epidemiológicos: edad, sexo, presencia de comorbilidad, lugar de procedencia, fecha de consulta, cuadro clínico de presentación (fiebre, tos, expectoración purulenta o hemoptoica, disnea, dolor torácico, confusión mental, escalofríos), exámenes de laboratorio básicos (recuento de leucocitos, proteína $\mathrm{C}$ reactiva y radiografía de tórax), tratamiento antimicrobiano empírico prescrito en la Unidad de Emergencia y la evolución en el seguimiento a los 30 días. Los pacientes fueron manejados con los esquemas antibióticos recomendados por la Sociedad Chilena de Enfermedades Respiratorias ${ }^{0}$ : a) NAC tipo I (menores de 60 años sin comorbilidad) fueron tratados con amoxicilina $750 \mathrm{mg}$ cada $8 \mathrm{~h} \mathrm{o}$ claritromicina $500 \mathrm{mg}$ cada $12 \mathrm{~h}$ durante 10 días y fueron controlados en el consultorio cercano a su hogar a los 7-10 días; b) NAC tipo II ( $\geq 60$ años 0 presencia de comorbilidad) fueron tratados con amoxicilina-ácido clavulánico 875/125 mg cada 12 h o levofloxacina $500 \mathrm{mg}$ cada $12 \mathrm{~h}$ durante 10 días y fueron controlados a las $72 \mathrm{~h}$ en la Unidad de Emergencia antes de derivarlos a su Consultorio de referencia. La guía clínica nacional no menciona el uso de levofloxacina en el manejo de la neumonía ambulatoria; sin embargo, las guías internacionales recomiendan su empleo basado en su amplio espectro de acción ${ }^{6}$. En todos los pacientes se realizó seguimiento del estado vital, aparición de complicaciones o necesidad de hospitalización hasta los 30 días de efectuado el diagnóstico. En los pacientes con evolución clínica desfavorable que requirieron hospitalización, se registró la fecha y motivo de la admisión al hospital, el resultado de los exámenes microbiológicos, el tratamiento antibiótico prescrito y la evolución en el hospital. El manejo clínico se consideró adecuado si el paciente estaba vivo, no presentaba complicaciones y no había sido hospitalizado dentro de los 30 días de efectuado el diagnóstico.

Estadística. Los resultados son expresados como valores promedio \pm desviación estándar para las

Tabla 1. C aracterísticas clínico-epidemiológicas de los pacientes con neumonía comunitaria ambulatoria en el Servicio de Salud Viña del M ar-Q uillota, V Región

\begin{tabular}{|c|c|c|c|}
\hline & NAC Tipo I & \multicolumn{2}{|c|}{ NAC Tipo II } \\
\hline $\mathrm{N}$ & 124 & \multicolumn{2}{|c|}{187} \\
\hline Edad (años) $(\mathrm{X} \pm \mathrm{DE})$ & $38,5 \pm 16,0$ & \multicolumn{2}{|c|}{$68,7 \pm 16,1$} \\
\hline 15-44 años & $(63 \%)$ & 18 & $(10 \%)$ \\
\hline 45-64 años & $(37 \%)$ & 41 & $(23 \%)$ \\
\hline$\geq 65$ años & $(0 \%)$ & 122 & $(67 \%)$ \\
\hline Sexo (M-F) & $71-53(57-43 \%)$ & $82-105$ & $(44-56 \%)$ \\
\hline \multicolumn{4}{|l|}{ Procedencia: } \\
\hline Quillota & $(33 \%)$ & 71 & $(38 \%)$ \\
\hline Viña del Mar & $23 \quad(19 \%)$ & 52 & $(28 \%)$ \\
\hline Limache & $28 \quad(23 \%)$ & 14 & $(7 \%)$ \\
\hline La Ligua & $(10 \%)$ & 12 & $(6 \%)$ \\
\hline Cabildo & $(4 \%)$ & 18 & $(10 \%)$ \\
\hline Quintero & $(4 \%)$ & 11 & $(6 \%)$ \\
\hline Villa Alemana & $(5 \%)$ & 4 & $(2 \%)$ \\
\hline Petorca & $(2 \%)$ & 5 & $(3 \%)$ \\
\hline \multicolumn{4}{|l|}{ Mes de consulta: } \\
\hline Enero-marzo & $(2 \%)$ & 5 & $(3 \%)$ \\
\hline Abril-junio & $(21 \%)$ & 49 & $(26 \%)$ \\
\hline Julio-septiembre & $(75 \%)$ & 120 & $(65 \%)$ \\
\hline Octubre-diciembre & $(2 \%)$ & 11 & $(6 \%)$ \\
\hline
\end{tabular}

Nota: M: masculino, F: femenino, NAC: neumonía adquirida en la comunidad. 
variables medidas en escala numérica y en porcentaje para las medidas en escala nominal. Las variables cualitativas (antecedentes clínico-radiográficos, evolución clínica y letalidad) fueron comparadas mediante la prueba de Chi cuadrado y las variables continuas con la prueba $t$ de Student. Las diferencias entre las variables fueron consideradas significativas con un valor de $p$ $<0,05$.

\section{Resultados}

Características generales de la población. Durante el período del estudio, se evaluaron 311 adultos inmunocompetentes con neumonía comunitaria, sin criterios de gravedad, atendidos en las Unidades de Emergencia de ocho centros asistenciales del Servicio de Salud Viña del Mar-Quillota de la V Región. Las características clínico-epidemiológicas son descritas en la Tabla 1. La edad promedio fue $57 \pm 22$ años (R: $15-99), 49 \%$ eran varones y cerca de $40 \%$ correspondieron a adultos mayores de 65 años. El 73,6\% de los enfermos fue atendido en la Unidad de Emergencia de los Hospitales de Quillota, Viña del Mar o Limache, y 91,3\% de los casos se presentó durante los meses de otoño-invierno (Figura 1). La distribución estacional de los pacientes con neumonía comunitaria atendidos en las Unidades de Emergencia de la V Región, siguió un patrón similar a los casos de influenza notificados por los centros de vigilancia epidemiológica (consultorios №2, Miraflores y Quillota) $)^{5}$. El 40\% de los pacientes correspondió a la categoría de riesgo I y el $60 \%$ a la categoría de riesgo II de la SER ${ }^{8}$. En la categoría de menor riesgo, la edad promedio de los enfermos fue $38 \pm 16,0$ años, $57 \%$ eran varones, la mayoría fueron atendidos en los hospitales de Quillota, Limache o Viña del Mar (75\%) y sólo 3,7\% tenía NAC multilobar. En la categonía de mayor

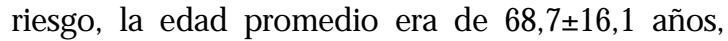
$44 \%$ eran varones, $76 \%$ fueron atendidos en los hospitales de Quillota, Viña del Mar o Cabildo, y 13,7\% tenían NAC multilobar. El cuadro clínico de presentación, el predominio de consultas en el período de otoño-invierno y los exámenes de laboratorio básicos (recuento de leucocitos y proteína $\mathrm{C}$ reactiva) fueron similares en ambas categonías de riesgo (Tablas 1 y 2).

Manejo de la neumonía en la Unidad de Emergencia. Los pacientes fueron evaluados por los médicos de la Unidad de Emergencia, quienes establecieron el diagnóstico y decidieron el lugar de manejo según las categorías de riesgo. Los pacientes con NAC tipo I fueron manejados con claritromicina $(71,8 \%) 0$ amoxicilina (26,6\%), y los pacientes con NAC tipo II fueron manejados con amoxicilina-ácido clavulánico $(80,7 \%)$ o levofloxacina (18,2\%). Todos los pacientes con NAC tipo I evolucionaron favorablemente desde

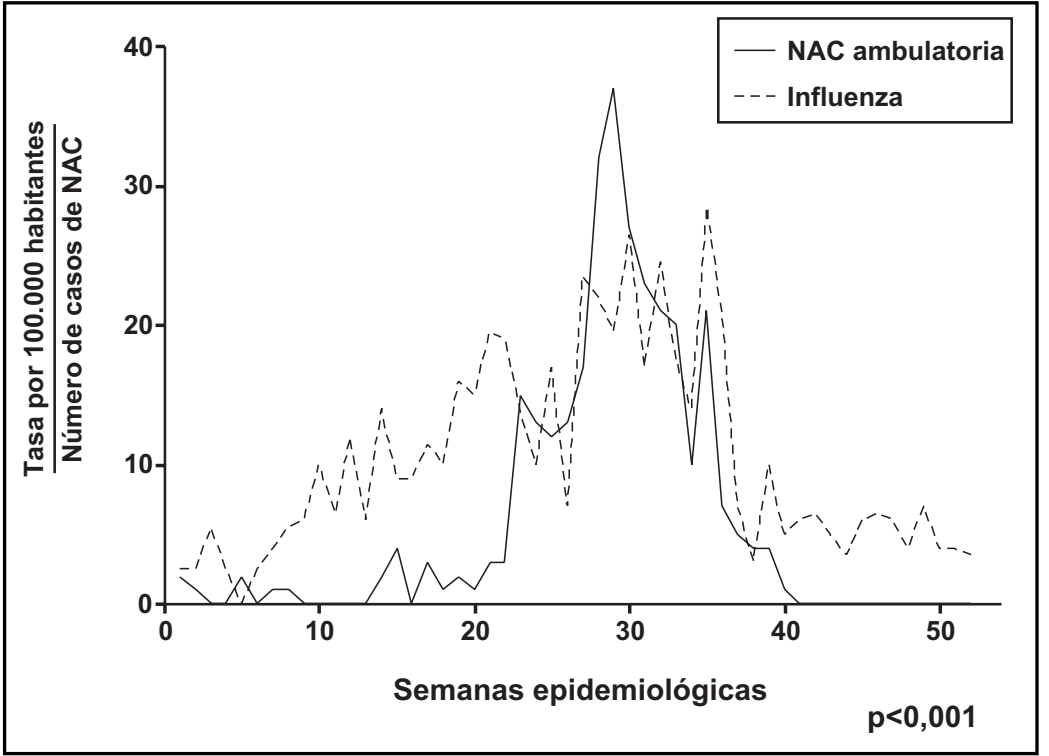

Figura 1. Distribución anual de los pacientes atendidos por neumonía comunitaria en la Unidad de Emergencia (casos) y tasa de notificación de influenza en el Servicio de Salud Viña del Mar-Quillota de la V Región (tasa x 100.000 hab) ${ }^{5}$. Enerodiciembre 2003. 
el punto de vista clínico y ninguno requirió ser admitido al hospital por la aparición de alguna complicación en la evolución. En la Tabla 3 se consigna la evolución de los síntomas respiratorios a las $72 \mathrm{~h}$ en los pacientes controlados en la Unidad de Emergencia con NAC tipo II. En la mayonía de los enfermos había cedido la fiebre pero persistía la tos y expectoración a las $72 \mathrm{~h}$ de seguimiento; en dos tercios de los casos habían disminuido los infiltrados radiográficos, sin embargo, en un tercio de los pacientes la radiografía de tórax no se había modificado (17,6\%) o los infiltrados pulmonares habían aumentado (16,2\%). Ninguno de los pacientes controlados a las $72 \mathrm{~h}$ en la Unidad de Emergencia presentaba deterioro clínico-radiográfico significativo que justificara su admisión al hospital.

\section{Tabla 2. C aracterísticas clínicas de los pacientes con neumonía comunitaria ambulatoria en el Servicio de Salud Viña del M ar-Q uillota, V Región}

\begin{tabular}{|c|c|c|}
\hline & NAC Tipo I & NAC Tipo II \\
\hline Fiebre & $95 \quad(76 \%)$ & $(56 \%)$ \\
\hline Tos & $116 \quad(94 \%)$ & $182 \quad(97 \%)$ \\
\hline Expectoración & $91 \quad(73 \%)$ & $156 \quad(83 \%)$ \\
\hline Dolor torácico & $87 \quad(70 \%)$ & $(59 \%)$ \\
\hline Leucocitos (por $\left.\mathrm{mm}^{3}\right)(\mathrm{X} \pm \mathrm{DE})$ & $12,633 \pm 5,074$ & $12,369 \pm 4,973$ \\
\hline Leucocitos $<4.000$ por $\mathrm{mm}^{3}$ & $2 / 80 \quad(2,5 \%)$ & $0 / 127$ \\
\hline Leucocitos $>12.000$ por $\mathrm{mm}^{3}$ & $(53 \%)$ & $60 / 127$ \\
\hline Proteína $C$ reactiva $(\mathrm{mg} / \mathrm{L})(\mathrm{X} \pm \mathrm{DE})$ & $157,3 \pm 124,4$ & $152,5 \pm 113,7$ \\
\hline Proteína $\mathrm{C}$ reactiva $\geq 10 \mathrm{mg} / \mathrm{L}$ & $(96 \%)$ & $81 / 81 \quad(100 \%)$ \\
\hline Proteína $\mathrm{C}$ reactiva $\geq 50 \mathrm{mg} / \mathrm{L}$ & $(83 \%)$ & $(83 \%)$ \\
\hline Neumonía multilobar & $4 / 109 \quad(3,7 \%)$ & $23 / 168(13,7 \%)$ \\
\hline
\end{tabular}

Nota: El número del denominador corresponde a los pacientes con la variable registrada.

Tabla 3. Tratamiento antimicrobiano prescrito y evolución clínica de la neumonía comunitaria ambulatoria del adulto en el Servicio de Salud Viña del M ar-Q uillota, V Región

Tratamiento antimicrobiano:

NAC Tipo I

Claritromicina

Amoxicilina

Eritromicina

NAC Tipo II

Amoxicilina-ácido clavulánico

Levofloxacina

Amoxicilina

Evolución clínica de la NAC Tipo II a las 72 horas:

Fiebre

Tos

Expectoración

Dolor torácico

Evolución clínica de la NAC ambulatoria:

Manejo clínico adecuado

Se hospitaliza

Fallece

$\begin{array}{rr}89 / 124 & (71,8 \%) \\ 33 / 124 & (26,6 \%) \\ 2 / 124 & (1,6 \%) \\ & \\ 151 / 187 & (80,7 \%) \\ 34 / 187 & (18,2 \%) \\ 2 / 187 & (1,1 \%)\end{array}$

$4 / 103$

$74 / 103$

$(71,8 \%)$

$53 / 103$

$(51,5 \%)$

$15 / 103$

$(14,6 \%)$

$308 / 311$

$3 / 311$

$3 / 311$
(99\%)

(1\%)

(1\%) 
Evolución clínica y mortalidad. De los 311 pacientes con NAC manejados inicialmente en el medio ambulatorio, en 308 pacientes (99\%) el manejo clínico fue adecuado y tres pacientes fueron admitidos al hospital debido a fracaso del tratamiento ambulatorio (1\%). Dos pacientes fallecieron en el hospital por sepsis y falla respiratoria progresiva, y otro falleció por descompensación cardiovascular, una semana después del egreso hospitalario (1\%). En la Tabla 4 se describe las características clínicas, tratamiento y evolución de los pacientes con NAC admitidos al hospital por fracaso en el manejo ambulatorio. Dos pacientes fallecidos fueron tratados con levofloxacina y uno con amoxicilina-ácido clavulánico en la Unidad de Emergencia del Hospital Gustavo Fricke, los tres decesos fueron atribuidos a la gravedad de la infección pulmonar, comorbilidad asociada o ambos, y correspondieron a episodios de neumonía comunitaria con criterios de gravedad que habrían justificado desde un comienzo su admisión y manejo en el hospital. Ninguno de los pacientes fallecidos acudió al control de las $72 \mathrm{~h}$ a la Unidad de Emergencia.

\section{DISCUSIÓN}

El principal objetivo del estudio fue examinar el manejo ambulatorio de la neumonía adquirida en la comunidad, en una cohorte de adultos inmunocompetentes atendidos en las Unidades de Emer- gencia del sistema público de nuestro país. Los principales hallazgos de este estudio fueron: 1) El manejo ambulatorio del paciente con neumonía comunitaria en los servicios de urgencia públicos de la V Región fue eficiente, con bajo riesgo de complicaciones y muerte; 2) El aumento estacional de las consultas por NAC durante el período de otoño-invierno coincidió con alta circulación de virus influenza en la población; 3) El tratamiento de la NAC ambulatoria con agentes ß-lactámicos y macrólidos tuvo similar eficacia clínica.

Se ha estimado que cerca de $80 \%$ de los episodios de NAC son manejados en el medio ambulatorio, debido a su bajo riesgo de complicaciones y letalidad inferior a $1-2 \% 10,11,15$. Sin embargo, los estudios nacionales que han evaluado este problema han sido escasos y, mayoritariamente, destinados a describir el manejo del paciente con NAC hospitalizado, quienes tienen mayor gravedad, riesgo de complicaciones y la letalidad de este grupo fluctúa entre 5 y $20 \% 16-22$.

En nuestro estudio, una elevada proporción de los pacientes con neumonía comunitaria eran ancianos o tenían alguna comorbilidad asociada, sin embargo no presentaban criterios de gravedad en la evaluación inicial, lo cual permitió que fueran manejados en el medio ambulatorio. La mayoría de los enfermos enrolados en el protocolo fueron atendidos en los hospitales de Quillota, Viña del Mar, Limache o La Ligua durante los meses de otoño-invierno (periodo de alta circula-

\section{Tabla 4. Características clínicas y evolución de los pacientes con neumonía comunitaria hospitalizados por fracaso del tratamiento ambulatorio}

\begin{tabular}{|c|c|c|c|c|c|c|}
\hline $\mathrm{N}$ & $\begin{array}{l}\text { Edad } \\
\text { (años) }\end{array}$ & Sexo & Comorbilidad & $\begin{array}{l}\text { Tratamiento } \\
\text { ambulatorio }\end{array}$ & $\begin{array}{l}\text { Tratamiento } \\
\text { en el hospital }\end{array}$ & Evolución \\
\hline 1 & 74 & $\mathrm{~F}$ & AVC-Anemia & Levofloxacina & Cefotaxima+CAF & Fallece por IRA \\
\hline 2 & 86 & M & Cardiopatía-EPOC & Levofloxacina & Cefotaxima & Fallece por ICC* \\
\hline 3 & 96 & $\mathrm{~F}$ & No & Amoxi-clavulanato & Cefotaxima & $\begin{array}{l}\text { Shock séptico-IRA } \\
\text { NAC aspirativa }\end{array}$ \\
\hline
\end{tabular}

Nota: M: masculino. F: femenino. AVC: accidente cerebrovascular con secuela motora. EPOC: enfermedad pulmonar obstructiva crónica tabáquica. CAF: cloranfenicol. IRA: insuficiencia respiratoria aguda. ICC: insuficiencia cardíaca congestiva. NAC: neumonía comunitaria.

*El paciente fallece por descompensación cardiovascular en el hogar una semana después del egreso hospitalario. 
ción de virus respiratorios en la población). Una vez establecido el diagnóstico clínico-radiográfico, los médicos de urgencia evaluaron la gravedad de la infección respiratoria y decidieron el manejo ambulatorio de los enfermos. En los pacientes menores de 60 años sin comorbilidad (NAC Tipo I), prescribieron agentes ß-lactámicos (amoxicilina) o macrólidos (claritromicina o eritromicina); en esta categoría, todos los pacientes evolucionaron favorablemente en el medio ambulatorio y no requirieron hospitalización. Los pacientes mayores de 60 años o con comorbilidad asociada (NAC Tipo II), fueron tratados mayoritariamente con agentes ß-lactámicos con inhibidor de betalactamasa (amoxicilina-ácido clavulánico) o fluoroquinolonas (levofloxacina). En esta categoría de riesgo, tres pacientes fueron admitidos al hospital debido a fracaso del tratamiento ambulatorio y fallecieron en el hospital o inmediatamente después del egreso hospitalario (letalidad: 1,6\%). Los antimicrobianos prescritos (ß-lactámicos, macrólidos y fluoroquinolonas), el riesgo de hospitalización y muerte de los pacientes con NAC inicialmente manejados en el medio ambulatorio fue similar a lo descrito en otros estudios ${ }^{23-26}$. Se ha estimado que entre $5 \%$ y $7,5 \%$ de los enfermos con NAC de bajo riesgo requerirá ser admitido al hospital en algún momento de la evolución debido a deterioro clínico progresivo o descompensación de alguna comorbilidad ${ }^{7,25}$.

Entre los pacientes con NAC Tipo II, $64 \%$ eran adultos mayores ( $>65$ años), la mayoría tenía enfermedades crónicas asociadas y en este grupo fue más frecuente la NAC multilobar; correspondiendo estas características clínicas a factores de riesgo de muerte o complicaciones previamente comunicados en estudios nacionales y extranjeros $^{1,6-10,20-22}$. Basados en estos antecedentes, el tratamiento antimicrobiano empírico prescrito y el control precoz en el servicio de urgencia 0 consultorio a las $72 \mathrm{~h}$ estarían plenamente justificados, ya que en esta categoría de riesgo se concentraron las complicaciones y los fracasos del tratamiento ambulatorio.

La guía clínica de la Sociedad de Tórax Americana ${ }^{6}$, recomienda el empleo de antibióticos ß-lactámicos asociado a macrólidos en los pacientes ambulatorios con comorbilidad cardiopulmo- nar y/o factores de riesgo de infección por $\mathrm{S}$ pneumoniae multirresistente; basado en estudios clínicos que sugieren que los pacientes tratados con macrólidos tendrían evolución clínica más favorable 27,28 . Sin embargo, en el presente estudio, el uso de agentes ß-lactámicos o macrólidos en el manejo de la NAC ambulatoria tuvo similar eficacia clínica, no observando diferencias significativas en la evolución clínica y el pronóstico de los enfermos según el agente antimicrobiano escogido. Si se considera la baja prevalencia de cepas de $\mathrm{S}$ pneumoniae multirresistentes en nuestra población ${ }^{29}$ y la falta de información local acerca de la importancia de los gérmenes atípi$\cos ^{16,19,30}$, los hallazgos del presente estudio permiten plantear que no existen fundamentos clínicos o bacteriológicos que sustenten el uso de terapia combinada de alto costo económico en nuestro medio. Además, en el Consenso de la Sociedad Chilena de Enfermedades Respiratorias se recomienda emplear macrólidos sólo en los pacientes hospitalizados con neumonía comunitaria grave en la UCI o sospecha de infección por gérmenes atípicos ${ }^{8}$.

En resumen, la neumonía comunitaria de manejo ambulatorio continúa siendo un importante problema de salud pública en nuestro país, con una alta demanda de recursos sanitarios, siendo su tasa de letalidad y complicaciones relativamente baja comparado con aquellos manejados en el hospital. En el presente estudio hemos comunicado que el manejo ambulatorio de la NAC del adulto, en los servicios de urgencia públicos de la V Región basado en las recomendaciones de la Sociedad Chilena de Enfermedades Respiratorias, ha resultado efectivo en términos de lograr la recuperación clínica de los enfermos con una baja tasa de hospitalizaciones y letalidad, similar a lo descrito en estudios extranjeros. El buen resultado clínico obtenido parece estar más relacionado con la adecuada evaluación de la gravedad del paciente individual, que realiza el médico de urgencia, que con el antimicrobiano específico escogido. Consideramos necesario continuar realizando estudios locales en el medio ambulatorio con el propósito de racionalizar la distribución de los recursos humanos y materiales para el adecuado manejo de la neumonía comunitaria en el país. 


\section{REFERENCIAS}

1. Harrison BDW, Farr BM, Pugh S, Selkon JB. Community-acquired pneumonia in adults in British hospitals in 1982-1983: a survey of aetiology, mortality, prognostic factors and outcome. Q J Med 1987; 62: 195-220.

2. Gifason PP, Kapoor WN, Stone RA, Lave JR, Obrosky DS, Schulz R et al. Medical outcomes and antimicrobial costs with the use of the American Thoracic Society guidelines for outpatients with community-acquired pneumonia. JAMA 1997; 278: 32-9.

3. CAmpBell DG. Overview of community acquired pneumonia: prognosis and clinical features. Med Clin North Am 1994; 78: 1035-48.

4. Pinner RW, Teutsch SM, Simonsen L, Klug LA, Graber JM, Clarke MJ et al. Trends in infectious diseases mortality in the United States. JAMA 1996; 275: 189-93.

5. Ministerio de Salud de Chile. Programa de Infecciones Respiratorias Agudas (IRA), Departamento de Estadísticas e Información de Salud, Ministerio de Salud de Chile, 2002. (http://deis.minsal.cl/ index.asp).

6. Niederman MS, Mandell LA, Anzueto A, Bass JB, BRoughton WA, CAMPBELL GD ET AL. Guidelines for the management of adults with communityacquired pneumonia. Diagnosis, assessment of severity, antimicrobial therapy, and prevention. Am J Respir Crit Care Med 2001; 163: 1730-54.

7. British Thoracic Society Standards of Care CommitTEE. British Thoracic Society guidelines for the management of community-acquired pneumonia in adults. Thorax 2001; 56 (Suppl IV): 1-64.

8. Sociedad Chilena de EnFermedades Respiratorias. Consenso Nacional en Neumonías Adquiridas en la Comunidad en Adultos y Niños. Rev Chil Enf Respir 1999; 15: 67-136.

9. BaRTLETT JG, Mundy LM. Community-acquired pneumonia. N Engl J Med 1995; 333: 1618-24.

10. Fine MJ, Smith MA, Carson CA, Mutha SS, Sankey SS, WeissFeld LA, Kapoor WN. Prognosis and outcomes of patients with community-acquired pneumonia. A meta-analysis. JAMA 1996; 275: 134-41.

11. Niederman MS, Mccombs JS, Unger AN, Kumar A, Popovian R. The cost of treating communityacquired pneumonia. Clin Ther 1998; 20: 820-37.
12. DEAN NC. Use of prognostic scoring and outcome assessment tools in the admission decision for community-acquired pneumonia. Clin Chest Med 1999; 20: 521-9.

13. Jin Y, Marrie TJ, Carriere KC, Predy G, Houston $C$, NESS K ET AL. Variation in management of community-acquired pneumonia requiring admission to Alberta, Canada hospitals. Epidemiol Infect 2003; 130: 41-51.

14. Fang GD, Fine M, Orloff J, Arisumi D, Yu VL, KAPOOR W ET AL. New emerging etiologies for community-acquired pneumonia with implications for therapy. A prospective multicenter study of 359 cases. Medicine (Baltimore) 1990; 69: 307-16

15. GARIBALDI RA. Epidemiology of community-acquired respiratory tract infections in adults: incidence, etiology and impact. Am J Med 1985; 78 (Suppl 6B): 32-7.

16. Trucco O, Vicencio M, Salamanca L, Ojeda A, Oyonarte M, Prado V. Participación de Legionella pneumophila en neumonía extrahospitalaria del adulto en Santiago. Rev Chil Infect 1993; 10: 8995.

17. Jiménez P, Saldías F, Meneses M, Silva ME, Wilson MG, Оттн L Diagnostic fiberoptic bronchoscopy in patients with community-acquired pneumonia. Comparison between bronchoalveolar lavage and telescoping plugged catheter cultures. Chest 1993; 103: 1023-7.

18. Saldías F, Blacutt M, Moreno R. Manejo del paciente con neumonía grave en ventilación mecánica: utilidad del lavado broncoalveolar. Rev Méd Chile 1996; 124: 950-8.

19. Lobos T, Saldías F, Cartagena C, Jover E, Alvarez M, Moreno R. Chlamydia pneumoniae en pacientes con neumonía adquirida en la comunidad en Santiago de Chile. Rev Méd Chile 1998; 126: 14839.

20. Saldías F, Mardónez JM, Marchesse M, Viviani $P$, Farías G, Díaz A. Neumonía adquirida en la comunidad en el adulto hospitalizado. Cuadro clínico y factores pronósticos. Rev Méd Chile 2002; 130: 1373-82.

21. Díaz A, Calvo M, O’Brien A, Farías G, Mardónez JM, SALDías F. Utilidad clínica de los hemocultivos en pacientes hospitalizados por neumonía adquirida en la comunidad. Rev Méd Chile 2002; 130: 993-1000. 
22. Cereceda J, Maturana R, Acevedo V, Aylwin M, FLORES M. Indice de gravedad en neumonía comunitaria hospitalizada. Rev Chil Enf Respir 2003; 19: 155-9.

23. Woodhead MA, MacFariane JT, McCracken JS, Rose DH, FINCH RG. Prospective study of the aetiology and outcome of pneumonia in the community. Lancet 1987; 1: 671-4.

24. Almirall J, Morato I, Riera F, Verdaguer A, Priu R, CoLL P ET AL. Incidence of community-acquired pneumonia and Chlamydia pneumoniae infection: a prospective multicentre study. Eur Respir J 1993; 6: 14-8.

25. Minogue MF, Coley CM, Fine MJ, Marrie TJ, Kapoor WN, Singer DE. Patients hospitalized after initial outpatient treatment for community-acquired pneumonia. Ann Emerg Med 1998; 31: 376-80.

26. WOODHEAD M. Management of pneumonia in the outpatient setting. Semin Respir Infect 1998; 13: 816.
27. Gleason PP, Meehan TP, Fine JM, Galusha DH, Fine MJ. Associations between initial antimicrobial therapy and medical outcomes for hospitalized elderly patients with pneumonia. Arch Intern Med 1999; 159: 2562-72.

28. Marrie TJ, Peeling RW, Fine MJ, Singer DE, Coley CM, KAPOOR WN. Ambulatory patients with community-acquired pneumonia: the frequency of atypical agents and clinical course. Am J Med 1996; 101: 508-15.

29. Saldías F, Flores LJ, Torres $\mathrm{C}$, García P, Díaz A. Susceptibilidad a antimicrobianos de Streptococcus pneumoniae en población infantil y adulta de Santiago. Período 1997-2003. Rev Méd Chile 2005; 133: 42-9.

30. Díaz A, Fuentes G, Couble B, Unibe R, Mercado G, Soza A ET AL. Etiología de la neumonía adquirida en la comunidad en adultos hospitalizados en Santiago, Chile: implicancias para las guías clínicas. Rev Chil Enf Respir 2005; 21: 23-32.

\section{Agradecimientos}

Los autores agradecen la valiosa colaboración del equipo de salud del Servicio de Salud Viña del MarQuillota que participó en el proyecto de neumonía comunitaria del adulto:

Dra. Miriam Alday - Hospital Geriátrico Paz de la Tarde - Limache.

Dra. Soledad Báez - Hospital de Limache.

Dra. Fabiola Castro - Hospital de La Ligua.

Dr. Rodrigo Cruz - Hospital de Peñablanca.

Dr. León Ovalle J. - Hospital Gustavo Fricke - Viña del Mar.

Dra. Jeanne Carla Palet - Hospital de Quintero.

Dra. Aída Pizarro - Hospital de Petorca.

Dr. Juan Rivera - Hospital de La Calera.

Dr. Carlos Tapia - Hospital de Cabildo.

Sra. Ángela Velásquez - Servicio de Estadística, Servicio de Salud Viña del Mar-Quillota.

E.U. Sylvia Morris - Programa ERA, Servicio de Salud Viña del Mar-Quillota. 